\title{
Qualitative Descriptions of Image in the Gaussian Scale-Space
}

\author{
Tomoya SAKAI and Atsushi IMIYA \\ Institute of Media and Information Technology, Chiba University, Japan
}

Received January 20, 2005; final version accepted April 20, 2005

\begin{abstract}
This article addresses qualitative descriptions of image which characterise hierarchy and configuration of image structure. The qualitative descriptions are derived from trajectories of stationary points in the Gaussian scale-space and the gradient field of the scale-space image, which are called respectively "stationary curves" and "figure field". The stationary curves and figure-field fluxes define the scale-space hierarchy, which is explicitly described as a single tree by introducing a point at infinity. The configuration of image structure at fixed scale is represented by a pseudograph which is obtained from the figure field. The Voronoi tessellation is also employed to extract boundaries of image segments.
\end{abstract}

\section{Introduction}

This paper focuses on trajectories of stationary points and gradient field of images in the Gaussian scale-space in order to investigate topological properties of image. The basic concept of the gradient field was introduced by Zhao and Iijima $[9,10]$. They called the gradient field "figure field". We develop the theory on figure field to acquire the qualitative descriptions of image such as hierarchy and configuration of image structure.

The Gaussian scale-space analysis of images goes back to Iijima [1-5], who introduced the linear diffusion equation as the fundamental equation of figures starting from four axioms on the image operations: (i) nonnegative intensity of figures imaged on the retina, (ii) linearity of image intensity, (iii) closedness under affine transformations, and (iv) associative (or semigroup) property. Later Otsu [6] established an elegant formalisation of linear scale-space theory employing Lie group theory. Zhao and Iijima extended Iijima's theory to extract hierarchical structure of images using singular points of topography of grayscale image in a linear scale space [9]. In 1983, Witkin published a paper on scalespace filtering, and pointed out the importance of the Gaussian-kernel filter in image processing [7]. In 1984, Koenderink pointed out the mathematical equivalence between the Gaussian-kernel filtering and the linear diffusion equation, or the partial differential equation of the parabolic type [8].

Our paper is a sequel of Zhao and Iijima for construction of hierarchical tree and graph derived from the Gaussian scale-space. The figure field reveals connectivity among points of interest in the scale space-extrema, saddle, singular points and a point at infinity. Trajectories and connectivity of these points define a hierarchical structure of an image. This hierarchy is described as a tree. The figure field also defines a pseudograph, which expresses the configuration of extrema at a fixed scale. The pseudograph shows combinatorial properties with respect to the scale. In this paper, we also introduce a Voronoi-tessellation-based segmentation of the Gaussian diffusion-filtered images. This metric allows us to extract dominant parts of images as the Voronoi regions. Combination of a tree, which expresses the hierarchical relations, and a series of graphs, which expresses configurations at fixed scales qualitatively describes topological structure of the image in the scale space.

\section{Basic Theory}

\subsection{Gaussian scale-space}

The Gaussian convolution of a given positive function $f(\boldsymbol{x})$, i.e. the intensity of grayscale image yields a one parameter family of real-valued functions $f(\boldsymbol{x}, \tau)$. The parameter $\tau \geq 0$ represents the scale, which is related to the width of the Gaussian function. The transformation of the original image $f(\boldsymbol{x})$ into the Gaussian blurred images $f(\boldsymbol{x}, \tau)$ satisfies some fundamental principles: linearity, translation invariance, isotropy and semi-group structure $[7,11,12,19]$. The function $f(\boldsymbol{x}, \tau)$ denotes the Gaussian scale-space image of the original $f(\boldsymbol{x})$, that is, $f(\boldsymbol{x}, 0)=f(\boldsymbol{x})$.

The Gaussian scale-space image $f(\boldsymbol{x}, \tau)$ is the solution to the linear diffusion equation

$$
\frac{\partial}{\partial \tau} f(x, \tau)=\Delta f(x, \tau), \quad f(x, 0)=f(x)
$$

\subsection{Stationary curve}

A remarkable feature of the image is a set of stationary points of the image function. We define the stationary points and stationary curves as follows.

Definition 1. Stationary points are defined as the points where the gradient vanishes, that is, 


$$
\{\boldsymbol{x} \mid \nabla f(\boldsymbol{x}, \tau)=\mathbf{0}\} .
$$

Definition 2. The stationary curves are the trajectories of stationary points in the scale space.

We denote the trajectories of the stationary points as $\boldsymbol{x}(\tau)$. Setting $\boldsymbol{H}$ to be the Hessian matrix of $f(\boldsymbol{x}, \tau)$, Zhao and Iijima [9] showed that the stationary curves are the solutions of the system of linear equations:

$$
\boldsymbol{H} \frac{d \boldsymbol{x}(\tau)}{d \tau}=-\nabla \Delta f(\boldsymbol{x}(\tau), \tau)
$$

They $[9,10]$ clarified the topological properties of the stationary curves in one- and two-dimensional patterns. Equation (3) is solvable for nonsingular points where the Hessian matrix is always regular. Equation (3) also indicates that the stationary points instantaneously have infinite velocity in the scale space at singular points.

The stationary points of the two-dimensional scale-space images are classified into three types; local maximum points, local minimum points and saddle points. The type of stationary point can be discriminated by the second derivative of $f(\boldsymbol{x}, \tau)$ except at the singular points where det $\boldsymbol{H}=0$, namely, the second derivative test. Since the directional derivative of $f(\boldsymbol{x}, \tau)$ in the direction of a unit vector $\boldsymbol{n}$ is calculated as

$$
\frac{d f}{d n}=n^{\top} \nabla f
$$

the second directional derivative of $f(x, \tau)$ can be witten in the quadratic form:

$$
D^{2}(\boldsymbol{n}):=\frac{d^{2} f}{d n^{2}}=\boldsymbol{n}^{\top} \nabla\left(\boldsymbol{n}^{\top} \nabla f\right)=\boldsymbol{n}^{\top} \boldsymbol{H} \boldsymbol{n} .
$$

Therefore, if the second directional derivative $D^{2}(\boldsymbol{n})$ is positive for any direction of $\boldsymbol{n}$, then $f(\boldsymbol{x}, \tau)$ is convex. Analogously, $f(\boldsymbol{x}, \tau)$ is concave for negative $D^{2}(\boldsymbol{n})$. The stationary point at the concave (convex) point is said to be the local maximum (minimum) point.

Equation (5) implies that eigenvalues of the Hessian matrix of $f(\boldsymbol{x}, \tau)$ at $\boldsymbol{x}(\tau)$ are extrema of $D^{2}(\boldsymbol{n})$, so that

$$
\lambda_{2} \leq D^{2}(\boldsymbol{n}) \leq \lambda_{1},
$$

where $\lambda_{1}$ and $\lambda_{2}$ are the two eigenvalues of $\boldsymbol{H}$. We denote the signs of the eigenvalues of the Hessian matrix as $(-,-)$, $(+,-)$ and $(+,+)$, which correspond to the local maximum point, the saddle point and the local minimum point, respectively. Note that the type of stationary curves is classified as maximum curve, minimum curve, and saddle curve according to the second directional derivation in the same fashion. ${ }^{1}$

The following proposition denotes the signs of the Laplacian of stationary points.

Proposition 1. The sign of the Laplacian $\Delta f$ is positive (negative) at the local minimum (maximum) points. The Laplacian may have both signs at the saddle points.

Proof. The Laplacian $\Delta f$ is obtained as the trace of Hessian matrix $\boldsymbol{H}$, that is,

$$
\Delta f=\operatorname{tr} \boldsymbol{H}=\operatorname{tr}\left(\boldsymbol{X} \boldsymbol{\Lambda} \boldsymbol{X}^{-1}\right)=\operatorname{tr} \boldsymbol{\Lambda}=\sum_{i} \lambda_{i},
$$

where $\boldsymbol{X}$ is the square matrix whose column vectors are eigenvectors of $\boldsymbol{H}$, and $\boldsymbol{\Lambda}$ is the diagonal matrix of eigenvalues $\lambda_{i}$. The sum of the eigenvalues is positive (negative) at the local minimum (maximum) points.

It is notable that the stationary points with zero Laplacian can be found only on the saddle curves. This indicates that the scale-space stationary points

$$
\{(\boldsymbol{x}, \tau) \mid \nabla f=\mathbf{0} \text { and } \partial f / \partial \tau=\Delta f=0\}
$$

are always spatial saddle points. The saddle point with zero Laplacian is known as the balanced-saddle [14] or scalespace saddle [15].

\subsection{Figure field}

We focus on the gradient field of the Gaussian scale-space image. The basic idea of the figure field and the following definitions were provided by Zhao and Iijima [10].

Definition 3. Figure field $\boldsymbol{F}$ is defined as the negative of the gradient vector field of the scale-space image, that is,

$$
\boldsymbol{F}=-\nabla f(\boldsymbol{x}, \tau) \text {. }
$$

\footnotetext{
${ }^{1}$ In a number of papers related to the scale-space analysis, the stationary point and the stationary curve we define are called "critical point" and "critical curve". In the mathematical terminology "critical point" means the stationary points where $\nabla f=\mathbf{0}$ or the partial derivative of $f(\boldsymbol{x}, \tau)$ is not defined.
} 
Definition 4. Figure flow curve is the directional flux curve of figure field.

We have the following properties on figure field in scale space.

Proposition 2. Let $C$ denote a simple closed curve which encircles an arbitrary region $S$ in the two-dimensional scale-space image $f(\boldsymbol{x}, \tau)$. The net outward flux of the figure field is the rate of total loss of image intensity in $S$ with respect to the scale.

Proof. The net outward flux is

$$
\oint_{C} \boldsymbol{F}^{\top} \boldsymbol{n} d C
$$

where $\boldsymbol{n}$ is the unit normal vector to the curve $C$. From (1), (9) and the Gauss theorem derives the relation

$$
\oint_{C} \boldsymbol{F}^{\top} \boldsymbol{n} d C=-\oint_{C} \nabla f^{\top} \boldsymbol{n} d C=-\int_{S} \nabla^{\top} \nabla f d S=-\frac{\partial}{\partial \tau} \int_{S} f d S .
$$

The last notation in (11) denotes the derivative of total loss of image intensity in the region $S$ with respect to the scale $\tau$.

The following differential notation is equivalent to the above proposition.

$$
\frac{\partial f}{\partial \tau}+\nabla^{\top} \boldsymbol{F}=0
$$

This notation is directly obtained from (1) and (9). Equation (12) is exactly the conservation law of image intensity. Therefore, the figure field $\boldsymbol{F}$ is considered as the current density flow of image intensity.

Proposition 3. The local maxima and local minima are start-points and end-points of the figure flow curves.

It is trivial that $\boldsymbol{F}=-\nabla f \neq 0$ at any point $\boldsymbol{x}$ in the vicinity of an extrema, and we can draw a figure flow curve which passes through the point $\boldsymbol{x}$ in the direction of $\boldsymbol{F}$ unless $\boldsymbol{x}$ is not the extrema itself. In the sense of the current density flow of image intensity, the local maxima, local minima and saddle points are sources, drains and confluences of the flow, respectively. The following proposition indicates that the net field flux from the scale-space saddle is zero.

Proposition 4. The scale space stationary point is a divergenceless point of the figure field.

Proof. The scale-space stationary point is the point where the spatial and scale derivatives of $f(\boldsymbol{x}, \tau)$ are zero. Therefore, the scale-space stationary point is the spatial stationary point at which the Laplacian vanishes since $\partial f / \partial \tau=\Delta f=0$. This mathematical property indicates $\nabla^{\top} \boldsymbol{F}=-\nabla^{\top} \nabla f=0$.

Proposition 4 implies that the scale-space stationary point, or the scale-space saddle, is a confluent point where inward and outward figure flow are balanced and totally cancelled out. Griffin [14] classified the saddle point into the ridge-like, trough-like and balanced saddle point according to the sign of the Laplacian. The ridge-like and trough-like saddle points are the confluent points where the net field flux is negative and positive, respectively.

\section{Hierarchy}

The purpose of this section is to clarify the hierarchy of image structure which is implicitly suggested by the Gaussian scale-space. We show that the stationary curves and figure field completely define the scale-space hierarchy, which is explicitly described in the form of a tree.

\subsection{Concept}

As the scale parameter increases, we can observe that the stationary points of diffused image move in the space. The number of stationary points decreases when the different types of stationary points meet and annihilated at a singular point, and only one maximum point remains at the coarsest scale. This behaviour of stationary points is described as the stationary curves in the scale space. The different types of stationary curves share the singular point as their endpoint. Some singular points are connected by the stationary curves to the other singular point in higher scale. Therefore, the stationary curves imply the hierarchical relationship between singular points across scale.

One may assume that the connectivity of singular points described above is expressed as a tree. The leaves of the tree are the stationary points at the finest scale. The nodes of the tree are the singular points. The branches represent the connections by the stationary curves. However, the singular point at which the stationary points are annihilated does not always have the connection to the singular point in higher scale. In order to determine the scale-space hierarchy and to describe the hierarchy by a single tree, it is essential to find the stationary points as the additional nodes of tree to which the annihilation points are connected.

Such connectivity of singular points was firstly investigated by Zhao and Iijima [9, 10]. They paid attention to the 
extremum curves in the scale space. For one-dimensional signals, they have found a rule that even pairs of the maximum point and minimum point are annihilated at a singular point with increasing scale. Furthermore, this annihilation point can be uniquely connected to another maximum point since the minimum point generally exists between two maximum points. Thus, the scale-space hierarchy of the one-dimensional signal can be completely determined by the extremum curves.

For higher dimensional images, situations to find the connections are not so obvious. In the scale space for twodimensional images, the saddle points should be taken into consideration to clarify the connectivity of stationary points. While the maximum and minimum points are geometric cues for the extraction of the dominant parts of the image function, the saddle points play important role in the following aspects:

- The saddle points appear between the extrema at a fixed scale. The saddle points are representative of ridges and troughs which connect local maximum points and local minimum points, respectively.

- The saddle points are always involved in annihilations and creations of the stationary points [14, 15]. In twodimensional case, an extremum point is annihilated when it comes across a saddle point at a singular point with increasing scale.

- Scale-space saddles $\{(\boldsymbol{x}, \tau) \mid \nabla f=\mathbf{0}$ and $\partial f / \partial \tau=0\}$ are generally on the saddle curves $[14,15]$. The scale-space saddles are the divergenceless points of the figure field, as discussed in the previous section.

The saddle points are confluent points of two inward and two outward figure flow curves, which are called separatrices. Griffin et al. [14] investigated the behaviour of separatrices with respect to the scale. They argued that the separatrices which link stationary points might disappear, shrink, and merge before and after the annihilation/creation events. Since the separatrices are specific figure flow curves related to saddle points, the figure flow curves provide detailed information about the connectivity among stationary points including the singular points at a fixed scale.

In the following subsections, we briefly deal with two-dimensional images to simplify the discussion. The principle we suggest in this section can be applied to higher dimensional cases. We firstly observe the figure flow curves between stationary points and singular points. Secondly, we introduce a local minimum at infinity in order to define consistently the scale-space hierarchy. Finally, we propose the scale-space tree based on the singularities.

\subsection{Figure flow curves around singular point}

Let us observe the annihilation events. Figure 1 shows images of blobs and corresponding stationary curves in the scale space. The blobs are in an isotropic Gaussian shape, and the centres of the blobs are distributed at vertices of isosceles triangle with two long equal sides. A singular point at $\tau=1195$ is the annihilation point without connection. The scale-space images at $\tau=1195$ is pictured in Fig. 1(c). We consider the figure flow curves starting and ending at the annihilation point. The annihilation point of maximum point and saddle point, which appears in this case of triangle with long equal sides, is called a shoe point $[14,16]$. The shoe point has outward figure flow curves, but only one figure flow curve penetrates into the shoe point along the "instep" of the shoe. This figure flow curve connects the shoe point to another maximum point, which can be regarded as a parent of the shoe point. Therefore, in the case of the triangle with long equal sides, the hierarchical structure can be described by a tree as shown in Fig. 2.

However, we cannot always identify the stationary point as the parent of annihilation point in the finite domain of image. Figure 3 shows a case of isosceles triangle with two short equal sides. We find an annihilation of minimum point and saddle point at $\tau=166$ (Fig. 3(c)). This annihilation point has inward figure flow curves, but only one outward figure flow curve is found. The outward figure flow curve reaches to the end of the region of image. This example suggests that the annihilation point is linked to a drain of whole image intensity.

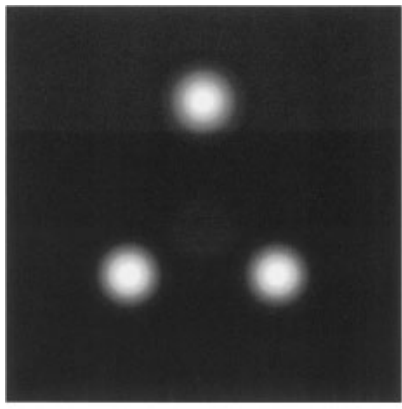

(a)

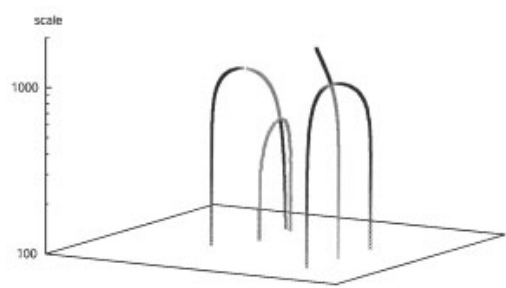

(b)

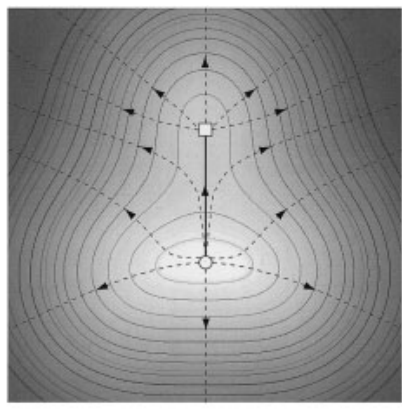

(c)

Fig. 1. Blobs at vertices of isosceles triangle with two long equal sides: (a) initial image, (b) stationary curves in $(x, y, \tau)$ scale space, and (c) the scale-space image at $\tau=1195$. Black and gray points in (b) correspond to extrema and saddles. Shoe point and local maximum point are indicated with square and circle in (c), respectively. 


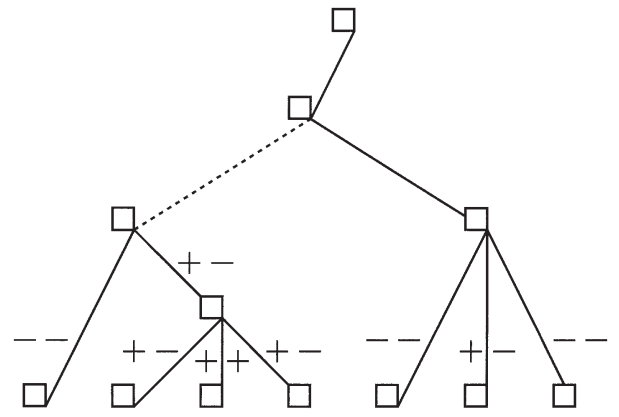

Fig. 2. Scale-space tree of Fig. 1(a). Solid lines and dotted line indicate the connections via stationary curves and figure flow curve, respectively.

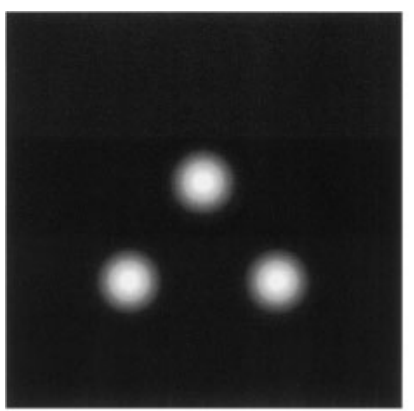

(a)

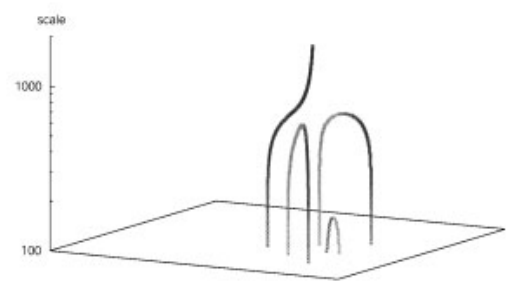

(b)

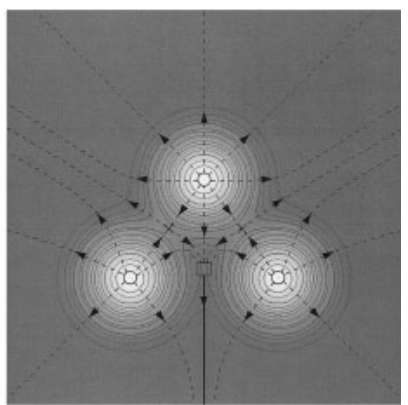

(c)

Fig. 3. Same as Fig. 1 but for an image of blobs at vertices of isosceles triangle with two short equal sides. The annihilation point of local minimum and saddle at $\tau=166$ is indicated with square in (c).

\subsection{Stationary point at infinity}

We conclude that the annihilation point of the local minimum in Fig. 3 is connected to a point at infinity. If the image function $f(\boldsymbol{x}, \tau)$ is defined in the infinite domain, all of the outward figure flow curves from the whole region of the image are considered to converge at the point at infinity. Since the image function $f(\boldsymbol{x}, \tau)$ is positive, the point at infinity is a drain, that is, the local minimum point. This local minimum point at infinity is representative of the dark background of the positive image. The local minimum point at infinity resides throughout the scale. We define the collection of local minimum points at infinity as the local minimum curve at infinity.

Furthermore, we presume that the local minimum point at infinity is annihilated with one remaining maximum point at infinite scale. This concept allows us to connect the remaining maximum curve to the local minimum curve at infinity. Consequently, the annihilation point is connected to the remaining maximum curve through the figure flow curve and the local minimum curve at infinity.

\subsection{Scale-space tree}

The hierarchical structure of image is described by a tree. The root of the tree is a virtual annihilation point of the local minimum point at infinity and remaining maximum point at infinite scale. The nodes of the tree are singular points. Stationary points which are connected to the annihilation points by the figure flow curves are also selected as the nodes of the tree. Some nodes may be the points at infinity. The leaves of the tree are the stationary points at the finest scale including the local minimum at infinity. The branches indicate the connections between the nodes by the stationary curves in the scale space and the figure flow curves. Thus, the figure field and stationary curves define the scale-space hierarchy. Figure 4 illustrates the construction of tree for the image in Fig. 3(a).
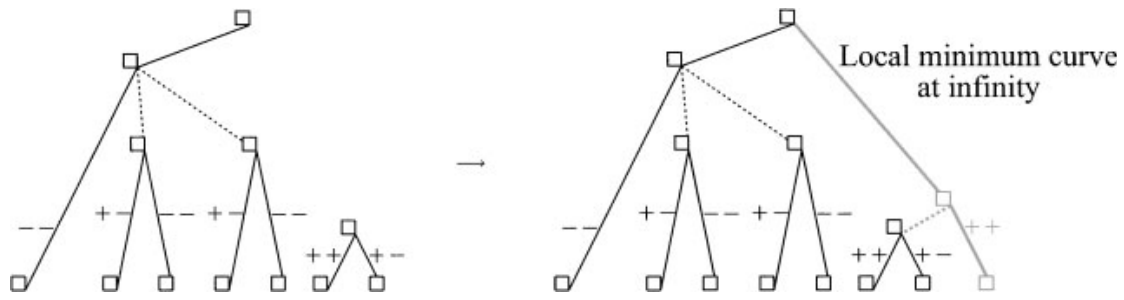

Fig. 4. Scale space tree of Fig. 3(a). The annihilation point of local minimum and saddle is linked to a local minimum point at infinity, which is annihilated with the remaining maximum point at infinite scale. 

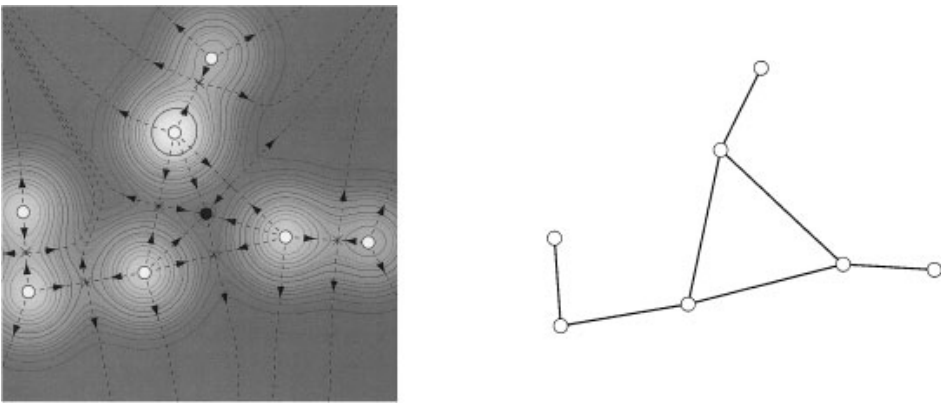

Fig. 5. Figure field and corresponding graph.

\section{Configuration of Structure}

Zhao and Iijima have proposed a graph description of the scale-space image at fixed scale [10]. The graph represents the configuration of extrema of the scale-space image $f(\boldsymbol{x}, \tau)$. Since two inward figure flow curves at a saddle point connect the sources of the flow curves, the saddle point is representative of a link of the sources. The sources of the figure flow are the local maximum points. Therefore, the local maximum point and the saddle point are regarded as the vertex and edge of the graph, respectively. The local minimum is the face of the graph. Thus, the graph is obtained from the figure field by the following rules:

1. Fix vertices at the local maxima.

2. Link the vertices with pairs of figure flow curves from saddle points to local maxima.

3. Remove the local minima and corresponding figure flow curves to generate faces.

Figure 5 illustrates the figure field and corresponding graph. Unless the image contains a singular point, we can uniquely derive the graph from the image at fixed scales.

Generally, the graph is simplified according to the diffusion of image $f(\boldsymbol{x}, \tau)$. The graph is simplified preserving its Euler's characteristic,

$$
\chi=V-E+F,
$$

where $V, E$ and $F$ denote the number of vertices, edges and faces of the graph, respectively. The Euler number $\chi=2$ when a point at infinity is taken into account. The point at infinity can be regarded as a local minimum for positive scale-space images. Note that the graph does not have edge-intersections. It is also worth noting that the graph may have multiple edges and self-loops, that is, the resulting graph is a pseudograph. A face inside a self-loop also represents a local minimum as shown in Fig. 6. An example of this self-loop can be found in Section 6.

\section{Segmentation}

In this section, we attempt to segment the scale-space image using the information concerning the extrema of the image. Since the image is blurred according to the scale, boundaries of the segment are not clearly detected by topographic approaches such as gradient-based edge detection. On the contrary, the positions of extrema are unambiguously determined at any scale. The extrema can be regarded as representatives of the segments. It is, therefore, reasonable to find the segmentation boundaries based on influence regions corresponding to the distributed extrema.

Such segmentation is achieved using the Voronoi tessellation. We regard the extrema in the scale-space image as the Voronoi generators. Each local maximum point, which is the vertex of the graph, is the representative of the segment which corresponds to a dominant part of bright object in the scale-space image. The local minimum point, which is assigned to the face of the graph, represents a cavity in the image.
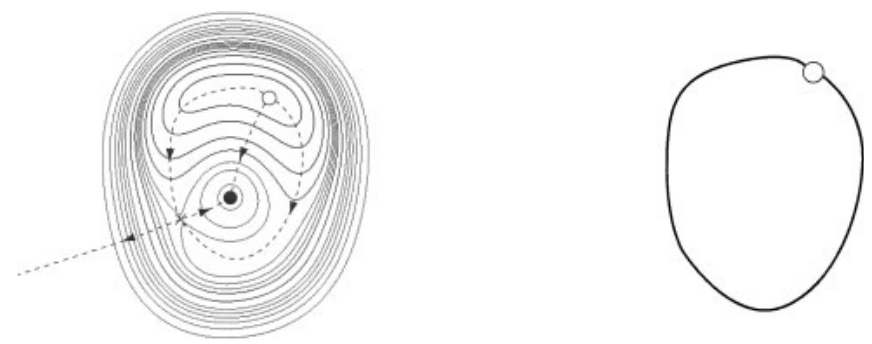

Fig. 6. Self-loop. 


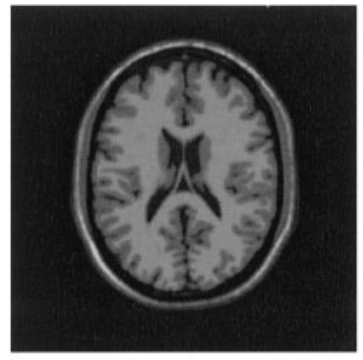

$\tau=0$

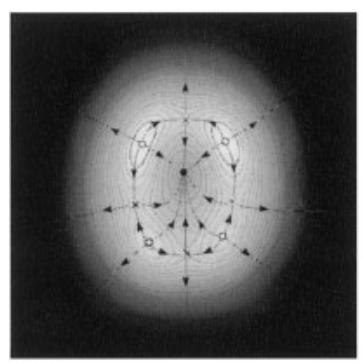

$\tau=30$

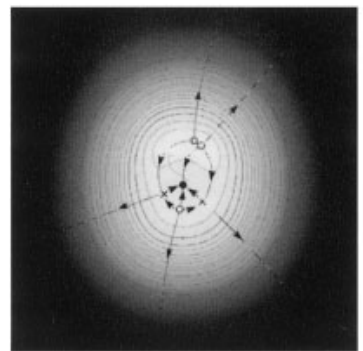

$\tau=89$

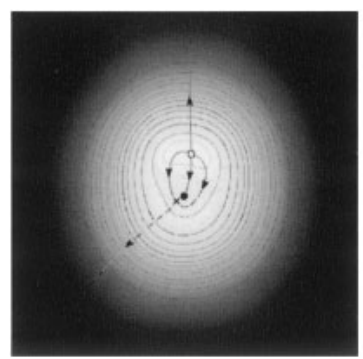

$\tau=96$

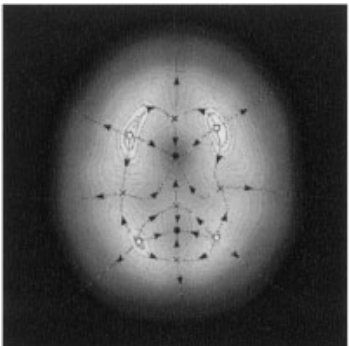

$\tau=10$

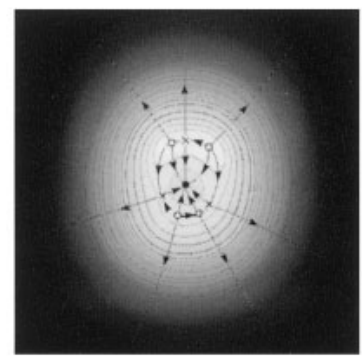

$\tau=85$

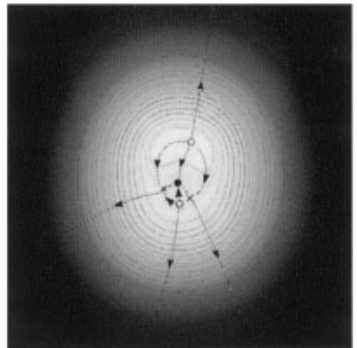

$\tau=93$

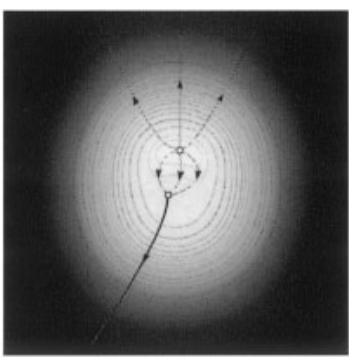

$\tau=97$

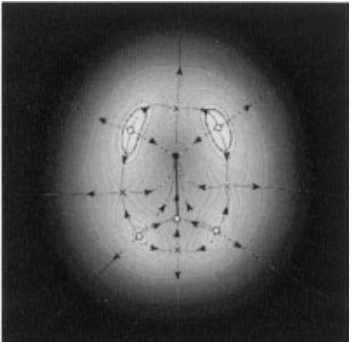

$\tau=19$

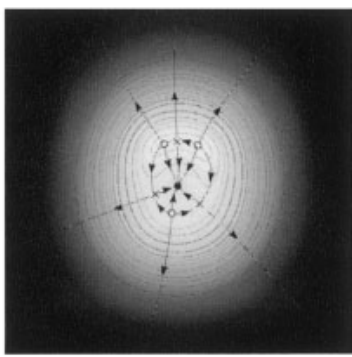

$\tau=87$

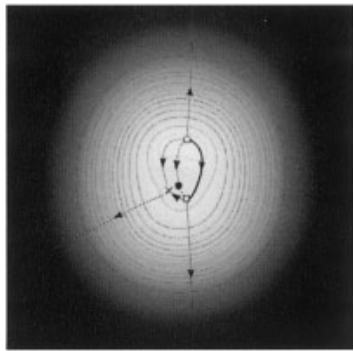

$\tau=95$

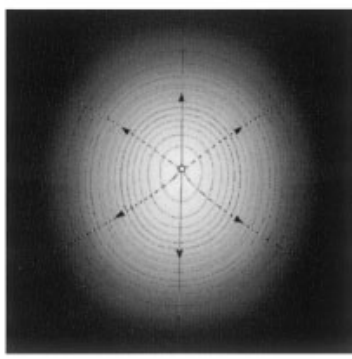

$\tau=125$

Fig. 7. Scale-space images of $256 \times 256$ MRI of brain, together with the figure flow curves. Top left panel shows the initial image. Scale increases from left to right, top to bottom. Maxima, minima and saddle points are indicated with open circles, filled circles and crosses, respectively. Annihilation points are plotted with open squares.

We also consider the metric in the linear scale-space. The linear scale-space is also called the Gaussian scale-space. Gaussian kernel with a deviation $\sqrt{2 \tau}$ is the Green's function of the linear diffusion equation of (1) at an infinite domain. Therefore, influence of an arbitrary point $\boldsymbol{p}$ on the point $\boldsymbol{x}$ is quantified by the Gaussian function; that is,

$$
\hat{f}(\boldsymbol{x}, \boldsymbol{p})=f \exp \left(-\frac{|\boldsymbol{x}-\boldsymbol{p}|^{2}}{4 \tau}\right)=\exp \left(-\frac{|\boldsymbol{x}-\boldsymbol{p}|^{2}}{4 \tau}+\ln f\right) .
$$

We define the Voronoi distance in the linear scale-space, using the exponent in (14) as,

$$
d\left(\boldsymbol{x}, \boldsymbol{p}_{n}\right)=\frac{\left|\boldsymbol{x}-\boldsymbol{p}_{n}\right|^{2}}{4 \tau_{n}}-\ln f_{n},
$$

where $\boldsymbol{p}_{n}$ is the position of Voronoi generator. In this paper, we assign $\tau_{n}$ in (15) as the scale of annihilation point corresponding to $\boldsymbol{p}_{n}$. Such scale is identified as the coarsest scale of the stationary curve to which the generator $\boldsymbol{p}_{n}$ belongs. The coefficient $f_{n}$ is selected as the intensity at the annihilation point. It is also possible to assign the scale 


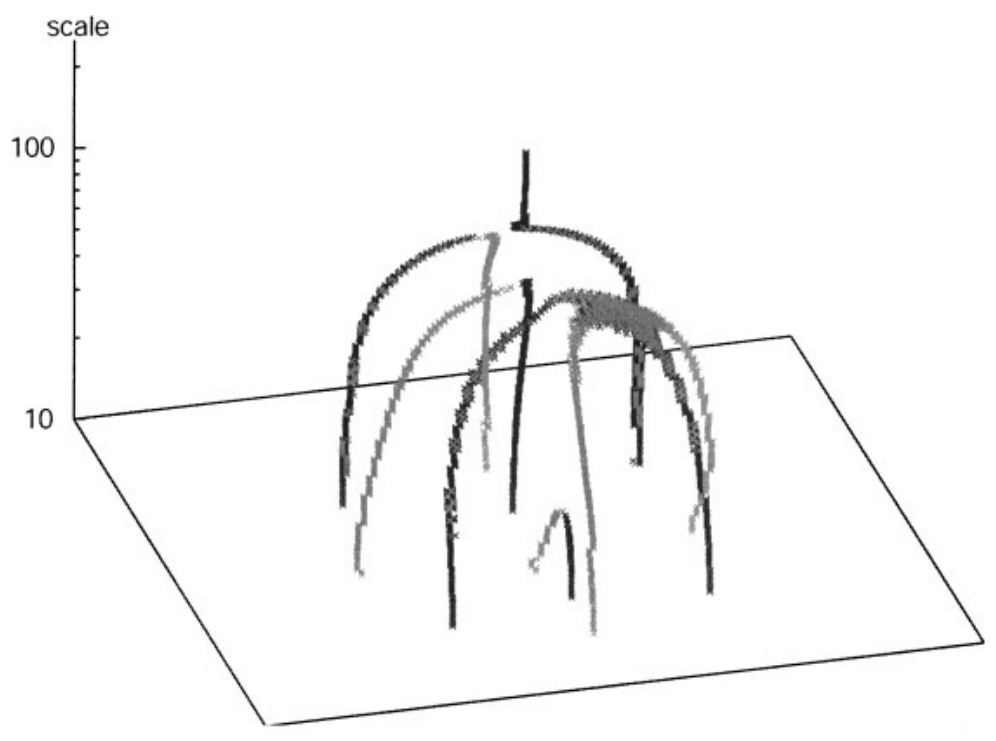

Fig. 8. Stationary curves in $(x, y, \tau)$ scale space of the artificial MRI brain.

where a scale-space saddle appears on the saddle curve associated with the extremum point $\boldsymbol{p}_{n}$, which is the same idea as [15].

The Voronoi distance (15) is similar to the power distance [18] or the compoundly weighted Voronoi distance [17] with the first and second weights being $4 \tau_{n}$ and $\ln f_{n}$, respectively.

The segmentation is performed by partitioning the space into the regions where all the interior points are closer to the corresponding Voronoi generator than to any other. The segments roughly estimate the shapes of objects and cavities corresponding to the extrema. Consequently, the scale-space image is qualitatively described as a set of the Voronoi segments.

\section{Numerical Example}

We demonstrate the extraction of scale-space hierarchy and the segmentation by the Voronoi diagram. As a twodimensional example, we take a slice of simulated MR brain image from the Brain Web [20]. Figure 7 shows 12 snapshots of diffusion process of the brain image together with the figure flow curves. The scale-space image at $\tau=10$ has 4 maxima, 2 minima (except a point at infinity) and 5 saddle points. Their trajectories in $(x, y, \tau)$ scale space are the stationary curves shown in Fig. 8. The stationary points are annihilated with increasing scale. The annihilation events occur at $\tau=19,85,90,95$ and 97 . The stationary curves describe the process of the annihilations.

The evolution of figure flow curves and corresponding graphs with respect to the scale are schematically illustrated in Fig. 9. The figure flow curves clarify the connections among stationary points at each scale. Here, we note again that a single inward (outward) figure flow curve is found at every annihilation point of local maximum (minimum) point and saddle point. This figure flow curve is indicated by a thick curve in Fig. 9 for each annihilation point. The single figure flow curve connects the annihilation point to the other extremum.

We see a self-loop in the scale-space image between $\tau=95$ and $\tau=97$. The local minimum point in the self-loop represents a dark internal region of brain image. This minimum point is related to a point at infinity, which represents the dark background of the image.

The resulting scale-space tree is shown in Fig. 10(a). The hierarchical structure is successfully determined by the figure field at each scale of annihilation and stationary curves over the scale space. It is confirmed that the local minimum curve at infinity is of the great importance in the construction of tree. Local minimum points in dark regions of the brain image are originally derived from the local minimum point at infinity. A subtree corresponding to the local minimum point at infinity describes how the dark regions in the brain image are derived from the dark background of the image.

Figure 10(b) shows an example of the Voronoi tessellation at $\tau=10$. The segmentation is achieved using the positions of the extrema and corresponding weights. The Voronoi segments approximate the shapes of dominant regions of the blurred image.

\section{Concluding Remarks}

Firstly, we showed that the figure field and stationary curves define the scale-space hierarchy. The stationary points have connectivity among them through the figure flow curves and the stationary curves in the scale space. Accounting a 

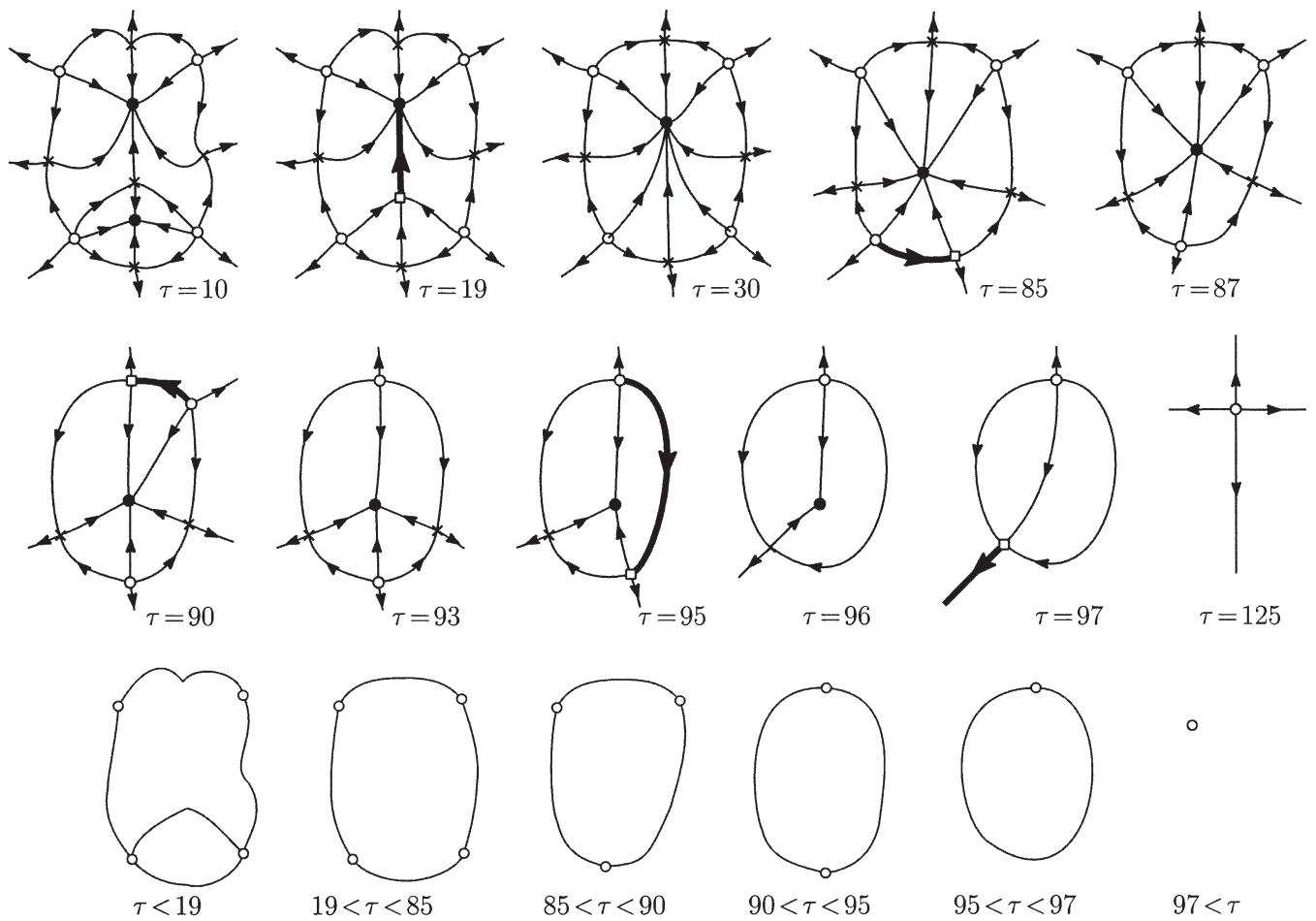

$85<\tau<90$

$90<\tau<95$

$95<\tau<97$

$97<\tau$

Fig. 9. Figure flow curves and graphs of the MR brain images. The first and second rows show the evolution of figure flow curves in increasing order of scale. Annihilation points are indicated with squares. The third row is a series of graphs.

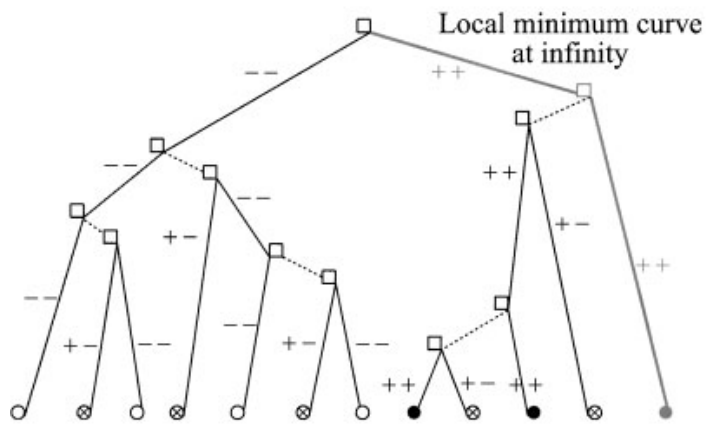

(a)

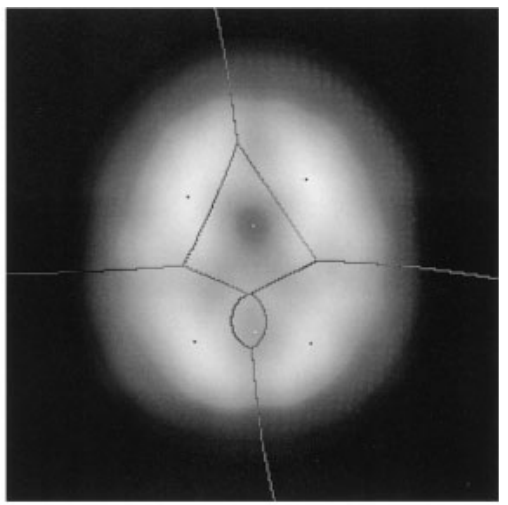

(b)

Fig. 10. Hierarchy and segmentation of the MRI of brain: (a) scale-space tree, and (b) scale-space image at $\tau=10$, together with Voronoi boundaries. Dots in (b) indicate the Voronoi generators.

point at infinity as a drain of the figure flow, we obtain a complete connectivity to determine the scale-space hierarchy. The hierarchical structure is described as a tree. Nodes of the tree are the singular points and the stationary points as parent nodes of annihilation points. Branches of the tree indicate the connections by the figure flow curves and the stationary curves in the scale space. The point at infinity is connected to a remaining maximum point at infinite scale.

Secondly, we describe the structure of scale-space image at fixed scale. The topological structure is expressed as a pseudograph. Vertices, edges and faces of the pseudograph correspond to the maximum points, saddle points and minimum points of the scale-space image, respectively. This pseudograph is obtained from the figure field at fixed scale.

We also achieved the segmentation of the scale-space image by the Voronoi tessellation, using the extrema as the Voronoi generators. The Voronoi distance is derived from the Gaussian kernel, which quantifies influence of an arbitrary point in the image. The Voronoi tessellation enables us to extract the boundaries of segments instead of edge detection, using only the geometric distribution of extrema and corresponding image intensities.

The boundary detection has been achieved by nonlinear scale-space analyses based on the gradient map [21, 22]. On the other hand, the Gaussian scale-space has advantage of constant diffusivity in the process giving rise to the space. Since the process of diffusion is linear and isotropic, the scale parameter uniquely defines the width of the Gaussian as a kernel function without regard to its position in the image. The scale-space image is composed of the Gaussian 
functions. Therefore, the configuration of the Gaussian functions qualitatively describes the image feature. The configuration is obtained from the stationary curves and figure field, as discussed in this paper.

\section{REFERENCES}

[1] Iijima, T., "Basic theory on the normalization of pattern (in case of typical one-dimensional pattern)," Bulletin of Electrotechnical Laboratory, 26: 368-388 (1962) (in Japanese).

[2] Iijima, T., "Basic theory on the normalization of two-dimensional visual pattern," Studies on Information and Control, Pattern Recognition Issue, IEICE Japan, 1: 15-22 (1963) (in Japanese).

[3] Iijima, T., "Basic equation of figure and observational transformations," IEICE Japan, Trans. C., 54-C: $37-38$ (1971).

[4] Iijima, T., Pattern Recognition, Corona Pub. Co. Ltd., Tokyo, (1973) (in Japanese).

[5] Iijima, T., The Fundamental Theory of Visual Information: The Foundation of the Pattern Recognition Problem, Corona Pub. Co. Ltd., Tokyo (1999).

[6] Otsu, N., "Mathematical Studies on Feature Extraction in Pattern Recognition," Researches of The Electrotechnical Laboratory, 818 (1981) (in Japanese).

[7] Witkin, A. P., "Scale space filtering," Proc. of 8th IJCAI, 1019-1022 (1983).

[8] Koenderink, J. J., "The structure of images," Biol. Cybern., 50: 363-370 (1984).

[9] Zhao, N.-Y., and Iijima, T., "Theory on the method of determination of view-point and field of vision during observation and measurement of figure," IEICE Japan, Trans. D., J68-D: 508-514 (1985) (in Japanese).

[10] Zhao, N.-Y., and Iijima, T., "A theory of feature extraction by the tree of stable view-points," IEICE Japan, Trans. D., J68-D: 1125-1135 (1985) (in Japanese).

[11] Weickert, J., Ishikawa, S., and Imiya, A., "Linear Scale-Space has First been Proposed in Japan,” Journal of Mathematical Imaging and Vision, 10: 237-252 (1999).

[12] Weickert, J., Ishikawa, S., and Imiya, A., "On the history of Gaussian scale-space axiomatics," Gaussian Scale-Space Theory, Vol. 8 of Computational Imaging and Vision Series, Kluwer Dordrecht, 45-59 (1997).

[13] Imiya, A., Sugiura, T., Sakai, T., and Kato, Y., "Temporal structure tree in digital linear scale space," LNCS, 2695: 356-371 (2003).

[14] Griffin, L. D., and Colchester, A., "Superficial and deep structure in linear diffusion scale space: Isophotes, critical points and separatrices," Image and Vision Computing, 13: 543-557 (1995).

[15] Kuijper, A., Florack, L. M. J., and Viergever, M. A., "Scale Space Hierarchy," Journal of Mathematical Imaging and Vision, 18-2: 169-189 (2003).

[16] Olsen, O. F., and Nielsen, M., "Generic events for the gradient squared with application to multi-scale segmentation," LNCS, 1252: 101-112 (1997).

[17] Okabe, A., Boots, B., and Sugihara, K., Spatial Tessellations-Concepts and Applications of Voronoi Diagrams, John Wiley and Sons (1992).

[18] Aurenhammer, F., and Klein, R., Voronoi Diagrams, In J.-R. Sack and J. Urrutia, editors, Handbook of Computational Geometry, Chapter 5, 201-290 Elsevier (2000).

[19] Lindeberg, T., Scale-Space Theory in Computer Vision, Kluwer, Boston (1994).

[20] Brain Web, http://www.bic.mni.mcgill.ca/brainweb/

[21] Perona, P., and Malik, J., "Scale space and edge detection using anisotropic diffusion," IEEE Trans. Pattern Anal. Mach. Intell., 12: 629-639 (1990).

[22] Weickert, J., Applications of nonlinear diffusion in Image Processing, Teubner, Stuttgart (1998).

\section{Appendix: A Equation of Stationary Curve}

A total differential equation of $\nabla f(\boldsymbol{x}, \tau)$ is written as:

$$
\frac{d}{d \tau} \nabla f=\boldsymbol{H} \frac{d \boldsymbol{x}}{d \tau}+\frac{\partial}{\partial \tau} \nabla f
$$

Since $\nabla$ and $\partial / \partial \tau$ are commutable, and $\nabla f=\mathbf{0}$ at the stationary points $\boldsymbol{x}(\tau)$, we have

$$
\boldsymbol{H} \frac{d \boldsymbol{x}(\tau)}{d \tau}=-\nabla \frac{\partial}{\partial \tau} f(\boldsymbol{x}(\tau), \tau) .
$$

Considering the governing equation (1) in the linear scale-space, we obtain (3) [9]. 